\title{
Modified Nitrogen-Doped Graphene Electrocatalyst for Oxygen Reduction Reaction in Alkaline Fuel Cells
}

\author{
Dureid Qazzazie $^{1,2}$, Michaela Beckert ${ }^{3}$, Rolf Mülhaupt ${ }^{3}$, Olena Yurchenko ${ }^{1,2}$ and Gerald Urban ${ }^{2}$ \\ 1. Freiburg Materials Research Center, University of Freiburg, Freiburg D-79104, Germany \\ 2. Laboratory for Sensors, Department of Microsystems Engineering, University of Freiburg, Freiburg D-79110, Germany \\ 3. Institute for Macromolecular Chemistry, University of Freiburg, Freiburg D-79104, Germany
}

Received: June 08, 2015 / Accepted: July 08, 2015 / Published: October 31, 2015.

\begin{abstract}
We report modified nitrogen-doped graphene $(\mathrm{CN})$ as electrocatalyst for ORR (oxygen reduction reaction) in alkaline medium. CN was synthesized by a novel procedure based on graphite oxide thermally treated with cyanamide suitable for facile $\mathrm{N}$-doping and large-scale production, whereas cyanamide was used as N-precursor. The structure of the material was characterized by TEM (transmission electron microscopy), SEM (scanning electron microscopy), Raman spectroscopy and XPS (X-ray photoelectron spectroscopy). Structural and electrochemical properties of CN were compared with those of non-modified graphene (TRGO (thermally reduced graphite oxide)). The electrochemical characterization of TRGO and CN in alkaline solution demonstrates enhanced electrocatalytic ORR activity and improved long-term stability for N-doped CN. Voltammetric studies confirmed that, oxygen reduction on $\mathrm{CN}$ rather follows four-electron pathway. Compared with commercial $20 \% \mathrm{PtC}$ catalyst, $\mathrm{CN}$ is characterized by exceptional methanol crossover resistance and superb long-term operation stability. Owing to these factors, nitrogen-doped graphene has a great potential to be used as metal-free electrocatalyst in cathodes of alkaline fuel cells.
\end{abstract}

Key words: Nitrogen-doped graphene, metal-free catalysis, oxygen reduction reaction, alkaline fuel cells.

\section{Introduction}

Fuel cells generate electric power through the conversion of chemical energy into electrical energy. Within the framework of an electrochemical process, the fuel such as hydrogen or methanol, oxidizes at the anode and the oxidizing agent, usually oxygen, is reduced to water at the cathode. In general, catalysts at the respective electrode are used to increase the fuel cells' efficiency.

Due to the sluggish ORR (oxygen reduction reaction) kinetics, platinum-based catalyst materials are widely investigated as cathodes for fuel cells to catalyse ORR in lab-scale applications. A fundamental drawback for the development of commercial fuel cells containing platinum-based electrodes is derived from high costs as

Corresponding author: Dureid Qazzazie, Ph.D. student, research fields: catalytic nanomaterials and fuel cells. E-mail: dureid.qazzazie@fmf.uni-freiburg.de. well as limited reserves of platinum in nature. Further problems arise from the vulnerability of platinum-based materials to time-dependent drift and poisoning effects, such as $\mathrm{CO}$ and alcohol "poisoning" leading to the catalyst deactivation $[1,2]$. Therefore, the development of novel metal-free materials has become the focus of research to substitute platinum-based catalysts. The performance requirements for alternative metal-free electrocatalysts in cathodes for the oxygen reduction reaction with regard to their electrocatalytic activity and stability were already reviewed many times [2, 3]. It becomes obvious that, the development of alternative lower cost electrocatalysts for the oxygen reduction reaction is of high significance for the further development of fuel cells and consequently, further progress towards clean energy generation.

According to their excellent properties, carbon-based materials, especially graphene have been 
moved into the focus of material research for electrochemical applications. Graphite and activated carbon already represent lower-cost alternative catalysts as cathodes for fuel cells in industrial applications. Compared with graphite and activated carbon, graphene is characterized by improved electrocatalytic ORR performance. Zhu, et al. [4] present in their review recent advancements in the development of different graphene-based electrocatalysts for ORR, highlighting specific graphene-based nanomaterials for electrochemical energy applications. Various modifications of graphene are of particular interest due to easy processability, low costs, large surface area and high porosity [5, 6], outstanding thermodynamic [7], electrical [8] and mechanical properties [9, 10]. All these exceptional properties also make graphene-based materials particularly feasible for use in micro energy harvesting applications. The electronic and optical properties of graphene can even be modified by doping with heteroatoms, such as nitrogen or boron [11, 12]. Hence, there is a growing trend towards doping graphene with heteroatoms to increase the electrocatalytic ORR performance, thus, producing modified Pt-free graphene-based electrocatalysts for ORR.

The focus of this paper is centered on nitrogen-doped graphene. The electrocatalytic ORR activity of nitrogen-containing carbon nanomaterials was originally reported in Refs. [11, 13, 14]. In their review, Wang, et al. [15] have summarized different synthesis and characterization methods of nitrogen-doped graphene. Based on experimental and theoretical studies, they also have presented recent applications of nitrogen-doped graphene. In particular, nitrogen-doped carbon-based materials represent promising metal-free electrocatalysts due to their enhanced electrocatalytic activity, long-term stability and environmental sustainability [16]. Compared with $\mathrm{Pt}$ and commercial PtC catalysts, nitrogen-doped graphene is characterized by notably better stability
[17, 18]. Besides, it is also demonstrated that, the activity of nitrogen-doped graphene is not influenced by adding carbon monoxide or methanol [11, 18].

In this context, it should be pointed out that, various techniques exist for graphene synthesis and modification. Micromechanical exfoliation has marked the breakthrough for the preparation of highly qualitative and pure graphene but the drawback here is low yield [19]. A further common method for the production of ultra-thin graphene with relatively high quality is the epitaxial growth which in turn suffers from high synthesis temperatures [20]. CVD (chemical vapor deposition) provides another common technique for the production of graphene capable for industrial applications [21]. However, prepared graphene by CVD suffers from impurity and the transfer of graphene to substrate can become complicated depending on the target substrate. Recently, Tölle, et al. [22] have developed a versatile method for a facile, low-cost, environmentally friendly and large-scale production of graphene-based materials offering a promising alternative to conventional techniques. The printing-based process features special opportunities for the integration of graphene-based materials in both macro-scale applications and, especially microsystems engineering as well as electronics applications.

Here, we report nitrogen-doped graphene as metal-free electrocatalyst for oxygen reduction reaction at alkaline conditions prepared by a novel synthesis procedure. The procedure is suited for facile production of nitrogen-doped graphene and based on graphite oxide thermally treated with cyanamide which is used as nitrogen-precursor. The structure of the prepared graphene-based materials was mainly characterized by Raman spectroscopy and XPS (X-ray photoelectron spectroscopy). Besides, graphene and nitrogen-doped graphene were electrochemically characterized by CV (cyclic voltammetry) and LSV (linear sweep voltammetry). Further electrochemical tests were conducted to investigate methanol crossover effect and long-term operation stability of prepared 
materials. The electrochemical properties and electrocatalytic activity of prepared graphene and nitrogen-doped graphene towards ORR are demonstrated and compared to those of commercial $20 \%$ $\mathrm{Pt} / \mathrm{C}$ catalyst. The use of nitrogen-doped graphene as alternative Pt-free electrocatalyst in alkaline fuel cells opens up opportunities for achieving clean and low-cost power generation.

\section{Experimental Details}

\subsection{Materials}

The graphene-based materials were synthesized by a process based on the thermal treatment of graphite oxide. A detailed report about the synthesis procedure is presented by Tschoppe, et al. [23]. Shortly, TRGO (thermally reduced graphite oxide) was synthesized by oxidation of graphite oxide and subsequent thermal reduction. Prepared graphene (TRGO) contains different oxygenated functional groups, mostly carboxylic and sulfonic acids. The nitrogen-doped derivative $(\mathrm{CN})$ was prepared by a similar procedure involving oxidation of graphite and subsequent thermal reduction in the presence of liquid cyanamide as a nitrogen-containing precursor, hence, enabling the incorporation of nitrogen atoms into carbon lattice. Both materials were prepared under identical thermal conditions.

\subsection{Surface Analysis}

TEM (transmission electron microscopy), SEM (scanning electron microscopy) and Raman spectroscopy were proceeded to evaluate the nanostructure and its morphology. The TEM-measurements were performed on a Leo 912-Omega transmission electron microscope of the company Zeiss (Oberkochen, Germany), with an acceleration voltage of $120 \mathrm{kV}$. The SEM images were taken using a Quanta (field emission gun) 250. The Raman measurements were performed at room temperature using Raman spectrometer (Confocal Raman MicrocopeLabram 800, JobinYvon Horiba) with a $632.8 \mathrm{~nm}$-wavelength laser (HeNe $20 \mathrm{MW}$ laser polarized 500:1). XPS was used to determine the elemental compositions of TRGO and $\mathrm{CN}$ and performed by XPS Perkin Elmer Phi 5600 System instrument with a $\mathrm{Mg}$-standard-anode as an excitation source (energy: $1,253.6 \mathrm{eV}$ ).

\subsection{Electrochemical Measurements}

The electrochemical characterization of TRGO and $\mathrm{CN}$ was performed via $\mathrm{CV}$ and LSV. The electrochemical measurements were carried out in a three electrode cell at a potential scan rate of $5 \mathrm{mV} \cdot \mathrm{s}^{-1}$ in alkaline $0.1 \mathrm{M}$ aqueous potassium hydroxide solution $(\mathrm{KOH})$. In order to prepare the electrode, $5 \mathrm{mg}$ of prepared TRGO-, CN powder or commercially available $\mathrm{Pt} / \mathrm{C}(20 \%$ wt. Pt) catalyst were dispersed in $5 \mathrm{~mL}$ isopropanol and sonicated for 30 min forming a homogeneous sample ink. The working electrode, a GC (glassy carbon) electrode of $3 \mathrm{~mm}$ in diameter, was subsequently coated with $20 \mu \mathrm{L}$ of the respective sample ink and dried in air for at least $24 \mathrm{~h}$. It may be pointed out that, neither Nafion nor any other solvent were added on top of electrode. A platinum sheet was used as counter electrode and $\mathrm{Ag} / \mathrm{AgCl}$ as reference electrode ( $3 \mathrm{M} \mathrm{KCl}$; double junction; Metrohm 6.0733.100). Before each measurement, the electrolyte either was degassed with nitrogen or oxygen gas for $20 \mathrm{~min}$, in order to saturate the solution. During the measurement, the gas flowing was maintained over the solution providing a continuous saturation of the electrolyte. All measurements were controlled using the potentiostat Autolab PGSTAT126N and the software GPES (general purpose electrochemical system) and were performed at room temperature (approximately $22^{\circ} \mathrm{C}$ ).

\section{Results and Discussions}

\subsection{Surface Characterization of TRGO and $C N$}

The surface morphology of prepared graphene-based materials was first investigated by TEM and SEM, both TEM and SEM images are exemplarily displayed 
in Fig. 1. The TEM image (Fig. 1a) confirms characteristic two dimensional (2D) structure of obtained few layer carbon nanosheets. The SEM image (Fig. 1b) illustrates the microporous structure of a typical nitrogen-doped graphene $(\mathrm{CN})$ sample. The prepared materials typically feature two-dimensional (2D) carbon nanosheets with tangly wrinkled morphology.

Raman spectroscopy was applied to investigate the defect level in carbon lattice of the prepared materials. Fig. 2a displays the Raman spectra of $\mathrm{CN}$ compared with TRGO and graphite. The Raman spectrum of graphite mainly shows a typical $\mathrm{G}$ peak at $1,576 \mathrm{~cm}^{-1}$ corresponding to ideal single-crystal graphite [24]. The Raman spectra of TRGO and CN display $G$ bands shifted to $1,595 \mathrm{~cm}^{-1}$ and $1,588 \mathrm{~cm}^{-1}$, respectively. The position of $\mathrm{G}$ band is quite sensitive to strains in the crystalline structure of carbon-based materials. The downshifted position of $\mathrm{G}$ band in graphite points to the relaxed graphitic structure of carbon lattice in graphite. Compared with TRGO the G peaks' position of $\mathrm{CN}$ shifts down closer to the $\mathrm{G}$ bands' position of graphite which we ascribe to the restoration of the graphitic structure in $\mathrm{CN}$ after the incorporation of nitrogen atoms and thermal treatment of the material [25].

Another predominant characteristic in the Raman spectra of prepared graphene- based materials is derived from the $\mathrm{D}$ band, usually represented by peaks at around $1,320-1,370 \mathrm{~cm}^{-1}$. In graphite, the $\mathrm{D}$ band is typically very weak as shown in Fig. 2a, while in contrast, clearly rised D bands appear at $1,344 \mathrm{~cm}^{-1}$ for TRGO and at $1,365 \mathrm{~cm}^{-1}$ for $\mathrm{CN}$. The $\mathrm{D}$ bands relate to the disorder-induced feature and their presence can be attributed to a modest defective structure of the graphene-based materials [26]. Besides, the $I_{D} / I_{G}$ ratio is taken into account to explore the density of defects in the samples. For graphite, the $I_{D} / I_{G}$ ratio is approximately $0.15, I_{D} / I_{G}$ increases to 1 for TRGO and 1.11 for $\mathrm{CN}$. The increase of $\mathrm{I} I_{D} / I_{G}$ can be ascribed to the presence of a substantial amount of defects in TRGO and CN. XPS was further used to characterize

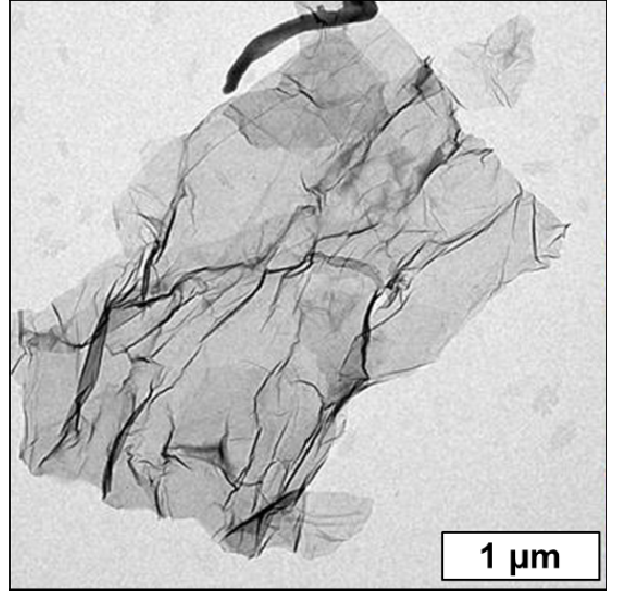

(a)

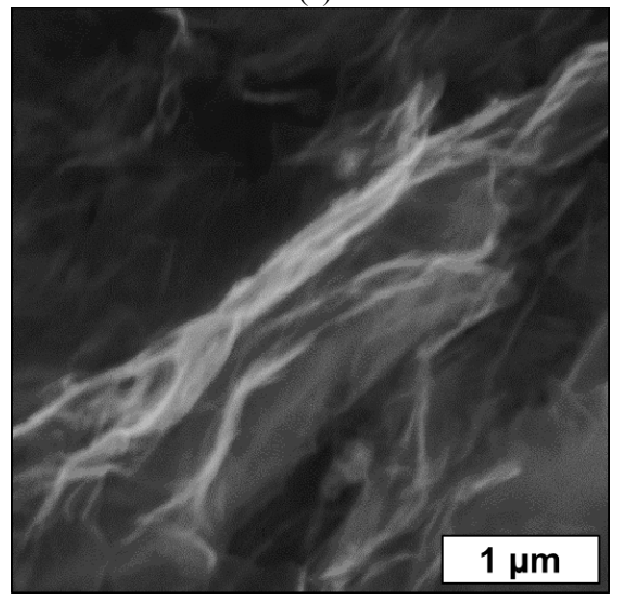

(b)

Fig. 1 (a) TEM image and (b) SEM image of typical nitrogen-doped graphene (CN) samples showing, the characteristic surface morphology of prepared materials.

the chemical composition of the prepared samples, thus, to verify the nitrogen-doping in carbon lattice. The XPS spectra of TRGO and CN, depicted in Fig. 2b, noticeably show major graphitic $\mathrm{C} 1 \mathrm{~s}$ peaks at about $285 \mathrm{eV}$. Oxygen functionalities are present in both TRGO and CN. Unlike TRGO, specific N1s peak occurs in the survey spectrum of $\mathrm{CN}$ at approximately $400 \mathrm{eV}$. The N1s peak corresponds to nitrogen and clearly confirms the successful incorporation of nitrogen atoms into carbon lattice of $\mathrm{CN}$. The peak intensity ratio of $\mathrm{N} 1 \mathrm{~s}, \mathrm{O} 1 \mathrm{~s}$ and $\mathrm{C} 1 \mathrm{~s}$ is used to identify the content of nitrogen in $\mathrm{CN}$, i.e., $11 \%$. The N1s spectrum were further deconvoluted to individual peaks, in order to determine the specific nitrogen configurations in $\mathrm{CN}$. The graphical illustration of high-resolution N1s XPS 


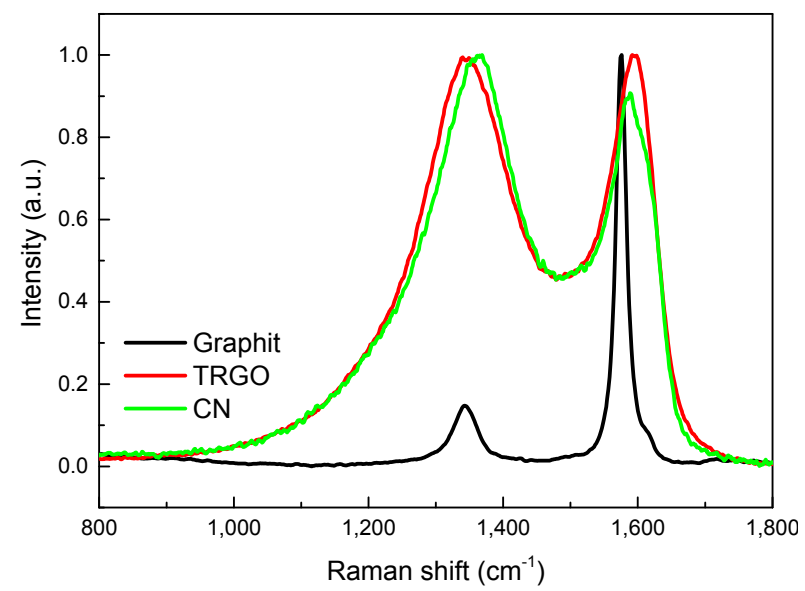

(a)

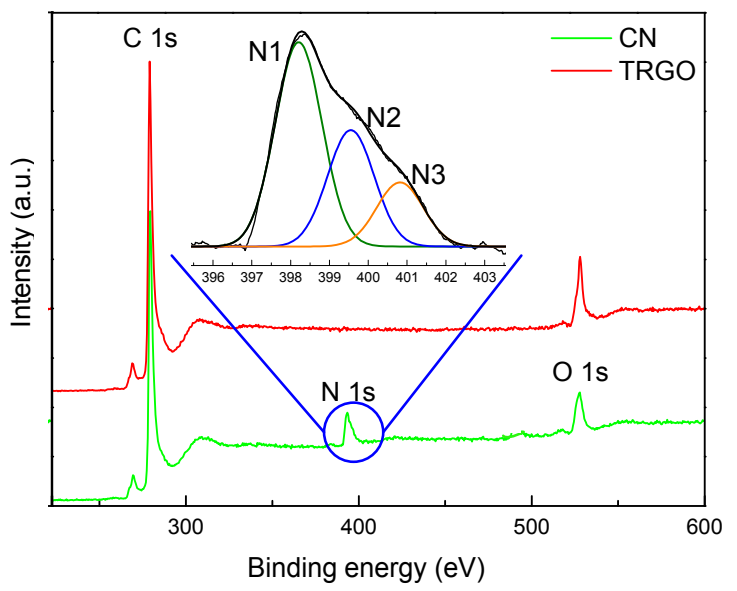

(b)

Fig. 2 Raman spectra of graphite, graphene (TRGO) and nitrogen-doped graphene (CN): (a) XPS spectra of grapheme (TRGO) andnitrogen-doped graphene (CN); (b) the inset in (b) shows high resolution N1s XPS spectra of CN and deconvolution into specific peaks corresponding to several nitrogen functionalities, $\mathrm{N} 1$ refers to pyridinic $\mathrm{N}$, N2 to pyrrolic $\mathrm{N}$ and $\mathrm{N} 3$ to graphitic $\mathrm{N}$, respectively.

spectra is presented in the inset in Fig. 2b. The high-resolution N1s peaks can be ascribed to pyridinic (N1), pyrrolic (N2) and graphitic (N3) nitrogen functionalities, determined at $398.2 \mathrm{eV}, 399.6 \mathrm{eV}$ and $400.8 \mathrm{eV}$, respectively. Hence, the certain amount of specific nitrogen functionality in total $\mathrm{N}$ atoms of $\mathrm{CN}$ for pyridinic N1, pyrrolic $\mathrm{N} 2$ and graphitic $\mathrm{N} 3$ was $54 \%, 30 \%$ and $16 \%$, respectively. The role of respective nitrogen functionalities in ORR process has been subject of controversial discussions about the active catalytic site in $\mathrm{N}$-doped carbon materials. Theoretical and experimental studies have been carried out to examine the ORR mechanism in N-doped carbon materials proposing pyridinic- $\mathrm{N}$ and graphitic- $\mathrm{N}$ as main catalytic active sites for ORR $[11,13,27,28]$.

\subsection{Electrochemical Characterization of TRGO and $\mathrm{CN}$}

The electrochemical properties of TRGO and $\mathrm{CN}$ were characterized via CV and LSV.

3.2.1 Electrocatalytic Reduction of Oxygen in Alkaline Solution

In order to confirm the electrocatalytic activity towards ORR, cyclic voltammetry and linear sweep voltammetry measurements were carried out in nitrogen- and oxygen-saturated alkaline electrolyte solution. CV measurements were first performed in nitrogen-saturated aqueous $0.1 \mathrm{M} \mathrm{KOH}$ solution. As shown in Fig. 3a, clear capacitive current background is identified within the CV. Afterwards, oxygen was introduced to the solution. The measured cyclic voltammograms, presented in Fig. $3 \mathrm{~b}$ feature specific cathodic current peaks, thus, demonstrating the ORR performance of all samples in oxygen-saturated $0.1 \mathrm{M}$ $\mathrm{KOH}$ solution. The highest value of current density among carbon-based materials is obtained for $\mathrm{CN}$ $\left(-0.256 \mathrm{~mA} \cdot \mathrm{cm}^{-2}\right)$, followed by TRGO $\left(0.175 \mathrm{~mA} \cdot \mathrm{cm}^{-2}\right)$ and GC $\left(0.150 \mathrm{~mA} \cdot \mathrm{cm}^{-2}\right)$. Compared with GC $(-250 \mathrm{mV})$, the onset potentials of TRGO $(-175 \mathrm{mV})$ and $\mathrm{CN}$ $(-160 \mathrm{mV})$ shift positively confirming better electrocatalytic activity towards ORR. Commercial PtC features positively shifted onset $(-20 \mathrm{mV})$ potential and higher current density $\left(0.285 \mathrm{~mA} \cdot \mathrm{cm}^{-2}\right)$ than $\mathrm{CN}$.

In addition, LSV measurements were performed on a $\mathrm{RDE}$ (rotating-disk electrode) in oxygen-saturated $0.1 \mathrm{M}$ $\mathrm{KOH}$ solution at a rotation rate of $1,600 \mathrm{rpm}$. The recorded linear sweep voltammograms, displayed in Fig. $3 \mathrm{c}$, show that, $\mathrm{CN}$ has the most positive onset potential and highest current density among the carbon-based materials. The LSV observations accord 


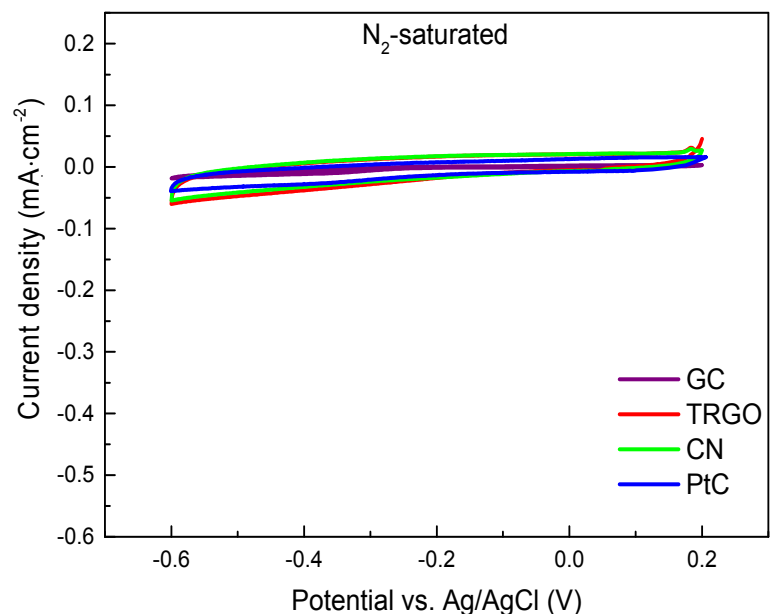

(a)

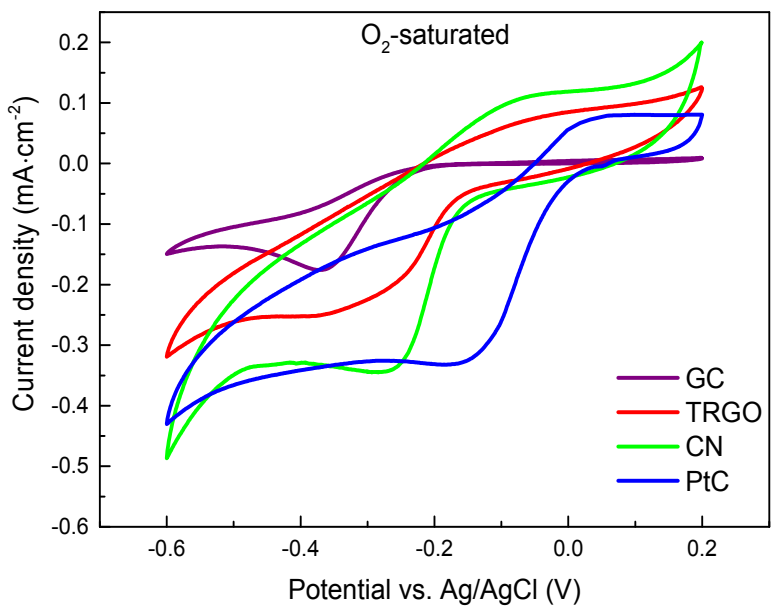

(b)

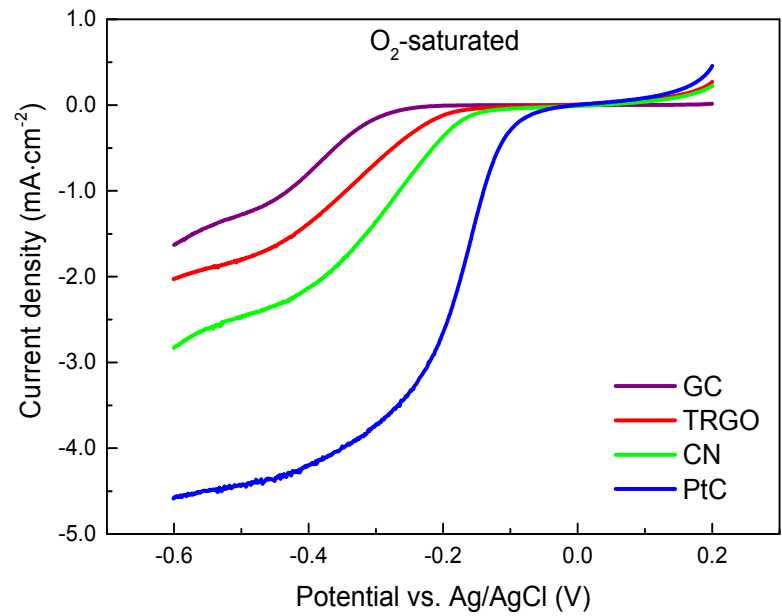

(c)

Fig. 3 CV-measurement of GC, TRGO, $\mathrm{CN}$ and commercial $20 \%$ PtC in aqueous $0.1 \mathrm{M}$ KOH solution in (a) $\mathrm{N}_{2}$-saturatedambience, and in (b) $\mathrm{O}_{2}$-saturated ambience demonstrating electrocatalytic activity towards ORR. LSV measurements of GC, TRGO, $\mathrm{CN}$ and $\mathrm{PtC}$ in $\mathrm{O}_{2}-(\mathrm{c})$ saturated $0.1 \mathrm{M} \mathrm{KOH}$ solution at rotation speed of $1,600 \mathrm{rpm}$.

with the $\mathrm{CV}$ results and demonstrate that, the electrocatalytic activity of graphene towards ORR is enhanced by doping with nitrogen. It should be noted that, TRGO contains different oxygenated functional groups, mostly carboxylic and sulfonic acids. Remaining oxygenated functional groups at TRGO do not contribute to better ORR performance, as shown by performed comparative ORR measurements for different TRGO samples prepared at identical treatment temperature, i.e., $600{ }^{\circ} \mathrm{C}$.

3.2.2 Mechanism of Oxygen Reduction on Nitrogen-Doped Graphene

The electron transfer mechanism of an electrocatalyst has to be investigated, to gain insight into the ORR kinetics. There are two possible oxygen reduction pathways in alkaline and acidic aqueous solutions, i.e., the direct four-electron pathway and the two-electron pathway, whereas hydrogen peroxide is produced as intermediate [29]. With regard to fuel cell applications, the efficient direct four-electron pathway proves to be the most beneficial due to the direct production of water. The investigation of the number of transferred electrons within oxygen reduction processes is also carried out at alkaline conditions. RDE measurements were performed at different rotation speeds from $400 \mathrm{rpm}$ to 2,025 rpm in oxygen-saturated 0.1 M KOH solution. The K-L (Koutecky-Levich) equation and K-L plots were applied to determine the number of the 
transferred electrons [30].

LSV curves of PtC and CN, shown in Figs. 4a and 4c, demonstrate increasing current density with higher rotation speeds which is caused by shorter diffusion distances at higher speeds. Figs. $4 \mathrm{~b}$ and $4 \mathrm{~d}$ present the K-L plots, applied to evaluate the electron transfer process at $\mathrm{N}$-doped graphene and $\mathrm{PtC}$. The relationship between $j^{-1}$ and $w^{-1 / 2}$ is clearly linear from $-0.4 \mathrm{~V}$ to $-0.8 \mathrm{~V}$. Hence, the number of transferred electrons $(n)$ at certain potentials was determined from the slope of the $\mathrm{K}-\mathrm{L}$ plots. As expected, the oxygen reduction on $\mathrm{PtC}$ is dominated by the direct four-electron pathway, since $n$ was calculated to be constantly 4.0 for the whole region between $-0.4 \mathrm{~V}$ and $-0.8 \mathrm{~V}$ (Fig. 4b). For CN1000, $n$ was calculated to be 3.1 at $-0.4 \mathrm{~V}$ and 3.9 at $-0.8 \mathrm{~V}$

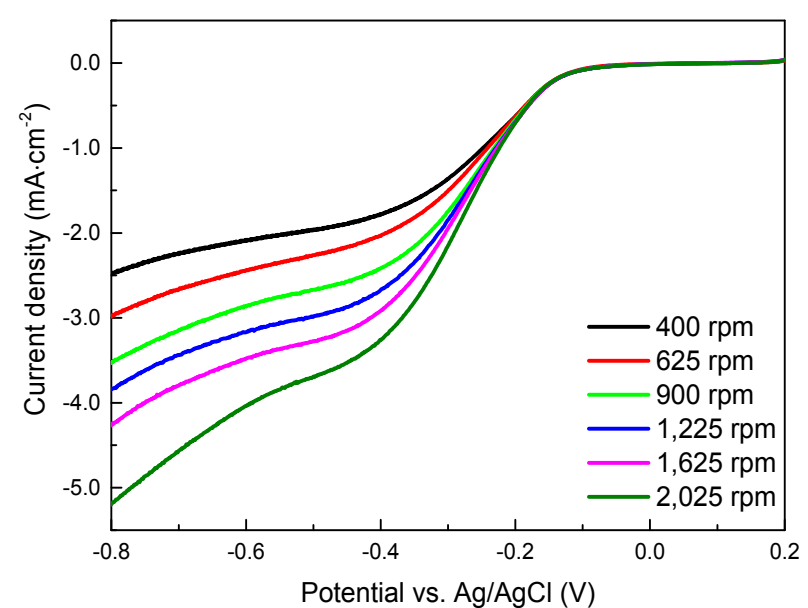

(a)

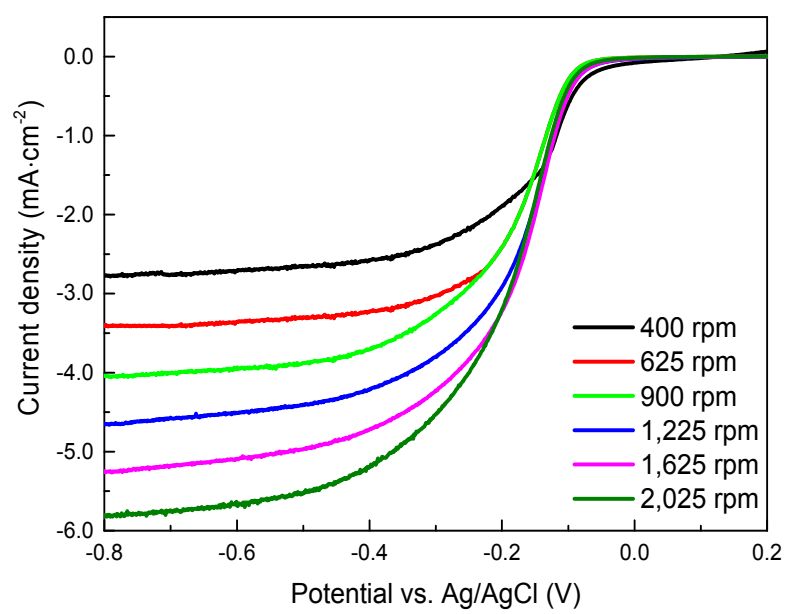

(c)
(Fig. 4d). It can therefore be concluded that, the oxygen reduction on $\mathrm{N}$-doped graphene at alkaline conditions involves both the two-electron pathway and the four-electron pathway. With increasing voltage the four-electron pathway is gaining in significance. The results imply that, the reduction process of oxygen on $\mathrm{N}$-doped graphene rather follows the four-electron transfer mechanism defined for alkaline conditions producing four $\mathrm{OH}^{-}$groups and not the transfer mechanism typical for acidic conditions where $\mathrm{H}^{+}$ participates in ORR [29].

3.2.3 Sensitivity towards Methanol Oxidation and Long-Term Operation Stability

Long-term stability and crossover effects play a crucial role in terms of fuel cell applications. In view of

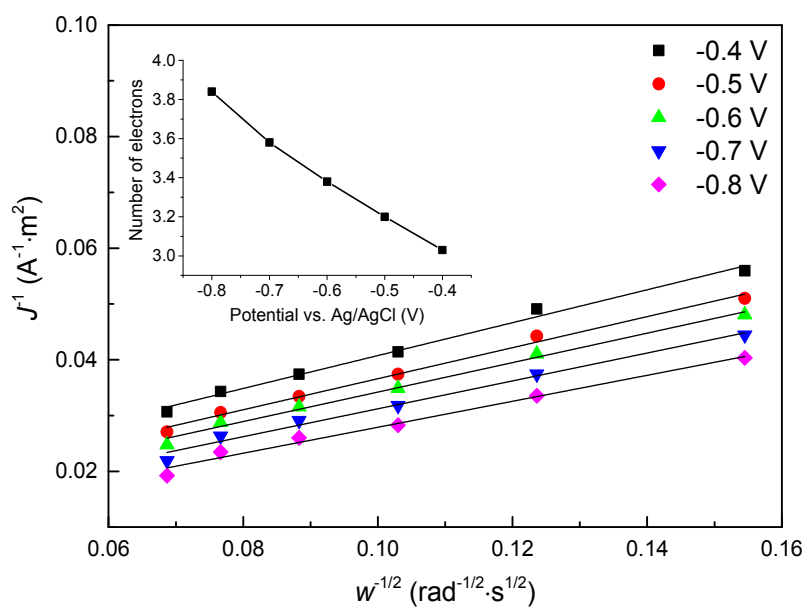

(b)

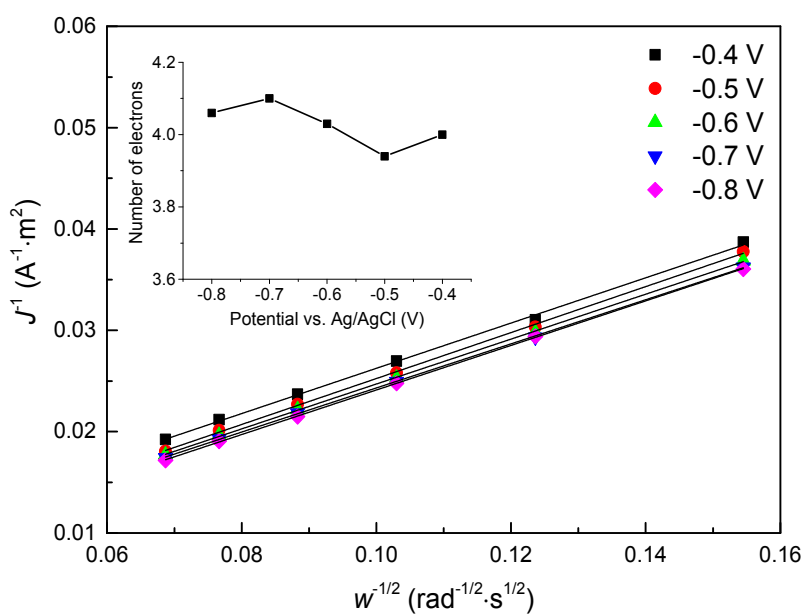

(d)

Fig. 4 Set of LSV curves for (a) CN and (c) $20 \%$ PtC recorded at alkaline conditions, i.e., in $\mathrm{O}_{2}$-saturated $0.1 \mathrm{M} \mathrm{KOH}$ solution at various rotation velocities with a scan rate of $5 \mathrm{mV} \cdot \mathrm{s}^{-1}$. K-L plots for (b) $\mathrm{CN1000}$ and (d) $20 \%$ PtC at different cathodic potentials. The numbers of transferred electrons at the respective potentials are shown in the insets in (b) and (d). 


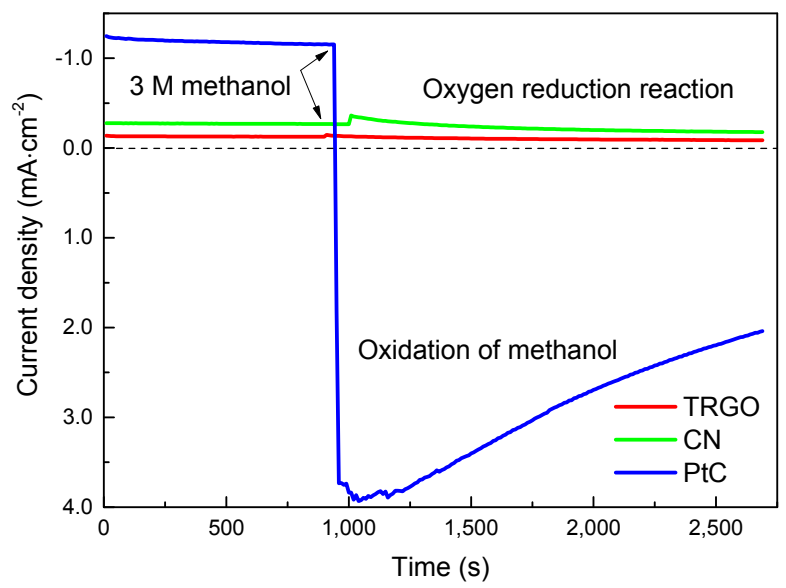

(a)

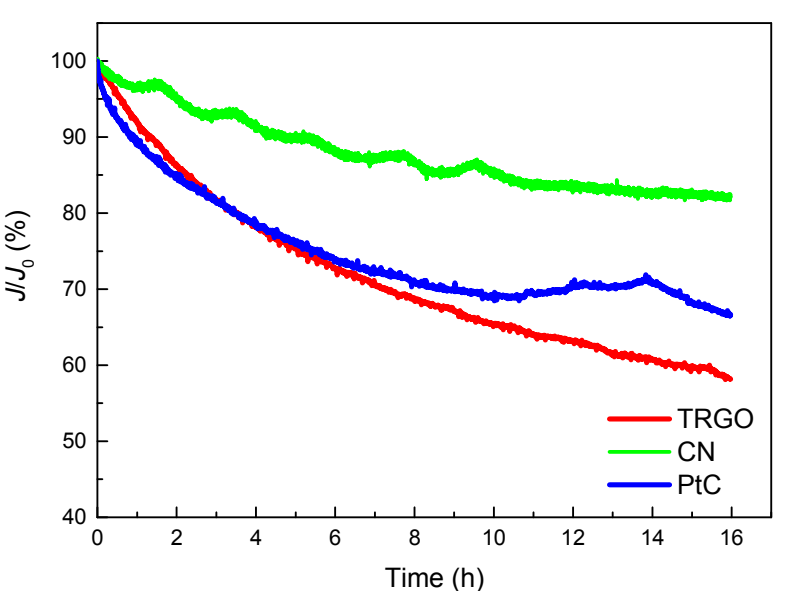

(b)

Fig. 5 ORR current-time responses at (a) TRGO, $\mathrm{CN}$ and PtC electrodes in $\mathrm{O}_{2}$-saturated $0.1 \mathrm{M} \mathrm{KOH}$ solution, before and after the addition of $3 \mathrm{M}$ methanol indicated by arrows. Long-term operation stability test, relative current-time responses for $16 \mathrm{~h}$ at (b) TRGO, CN and PtC electrodes in air-saturated $0.1 \mathrm{M} \mathrm{KOH}$ solution. All measurements are performed at rotation speed of $1,600 \mathrm{rpm}$.

a potential application in direct methanol alkaline fuel cells, prepared graphene and nitrogen-doped graphene were electrochemically characterized in presence of methanol as fuel molecule, in order to investigate a possible crossover effect. Moreover, the long-term stability of prepared materials in alkaline media was investigated. The long-term stability of TRGO and CN was preliminarily characterized by repetitive potential cycling, whereat $2,500 \mathrm{CV}$ cycles were performed consecutively between $-1.0 \mathrm{~V}$ and $0 \mathrm{~V}$ in $0.1 \mathrm{M} \mathrm{KOH}$, saturated with oxygen. After the repetitive potential cycling, the CVs have not shown any significant decrease in current and shape.

The electrocatalytic selectivity of TRGO and CN against the oxidation of methanol was characterized via chronoamperometry in oxygen-saturated $0.1 \mathrm{M} \mathrm{KOH}$ solution at $-0.25 \mathrm{~V}$ with the rotation rate of $1,600 \mathrm{rpm}$. The same procedure was applied for PtC catalyst due to comparison matters. The current-time chronoamperometric responses of TRGO, $\mathrm{CN}$ and $\mathrm{PtC}$ are displayed in Fig. 5a. After adding $3 \mathrm{M}$ methanol to the alkaline electrolyte solution, PtC suffers a keen positive shift of current density which can be attributed to the oxidation of methanol by PtC as shown in Fig. 5a. In contrast, the chronoamperometric responses of TRGO and $\mathrm{CN}$ change slightly upon the addition of $3 \mathrm{M}$ methanol and the oxygen reduction reaction at the respective graphene-based electrode remains stable. Thus, prepared TRGO and $\mathrm{CN}$ are characterized by excellent immunity against methanol crossover effect.

The long-term operation stability of prepared graphene-based materials and $\mathrm{PtC}$ was further characterized by the same chronoamperometric test over a longer period of time, whereas current-time response of each sample was recorded over $16 \mathrm{~h}$. The corresponding chronoamperometric responses of TRGO, $\mathrm{CN}$ and $\mathrm{PtC}$ are shown in Fig. 5b. A comparison of the current-time responses shows that, $\mathrm{CN}$ is characterized by best durability among investigated materials. After $16 \mathrm{~h}$, the relative current density of $\mathrm{CN}$ was still about $85 \%$, while, the relative current density of TRGO decreased to approximately $58 \%$ and the relative current density of $\mathrm{PtC}$ continuously decreased to about $66 \%$. These results suggest that, the incorporation of nitrogen atoms into carbon lattice enhances the long-term stability of graphene. The remaining oxygenated functional groups should be the cause for the operation instability of $\mathrm{TRGO}$ and $\mathrm{CN}$ in the long term. For $\mathrm{CN}$, the operation instability effect is still less remarkable, since mentioned functional groups barely contribute to ORR performance. Besides, the current density of $\mathrm{CN}$ was 
stabilized over time, while the current density of TRGO was gradually decreasing. It was already reported that, removing surface functional groups and oxygenated groups from the carbon surface improves stability of carbon-based electric double-layer capacitors [30]. Furthermore, the results indicate the insufficient long-term stability of $\mathrm{PtC}$ which is generally known for platinum-based materials.

Summing up, the addition of methanol has a negative impact on the performance of PtC shifting the negative cathodic peak related to ORR to positive current ascribed to electrooxidation of methanol. In contrast, TRGO and $\mathrm{CN}$ are remarkably immune against methanol crossover effect as compared to $\mathrm{PtC}$ in alkaline medium. Hence, methanol does not interfere with the oxygen reduction reaction at a potential graphene-based cathode. In terms of durability in alkaline medium, prepared CN clearly possesses better long-term operation stability than prepared TRGO and commercial PtC electrocatalyst. Again, the electrocatalytic ORR activity of $\mathrm{CN}$ is enhanced by nitrogen doping. Owing to these outstanding characteristics, prepared nitrogen-doped graphene by mentioned novel synthesis procedure is perfectly suitable for application as cathode material in direct methanol alkaline fuel cells.

\section{Conclusions}

In this paper, nitrogen-doped graphene was successfully prepared by novel synthesis procedure based on thermal reduction of graphite oxide and subsequent treatment with cyanamide. Specifically, the novel aspect of this work consists in the use of liquid cyanamide as nitrogen-precursor offering an easily manageable synthesis approach. We have determined by Raman spectroscopy a comparable defect density for both graphene and nitrogen-doped graphene. The distinct $\mathrm{D}$ bands in graphene and nitrogen-doped graphene indicate the high level of defects in prepared materials. The successful doping process of nitrogen in carbon lattice is evidenced by X-ray photoelectron spectroscopy. In particular, pyridinic, pyrollic and graphitic nitrogen functionalities were identified in carbon lattice of prepared nitrogen-doped graphene.

As shown by experimental results, prepared graphene and nitrogen-doped graphene have proven their electrocatalytic activity towards oxygen reduction reaction. Considered together with the results of structural characterization, we estimate a major importance of nitrogen doping for enhancing the ORR performance. Both graphene and nitrogen-doped graphene are highly resistant to methanol crossover. Nitrogen-doped graphene particularly exceeds commercial $20 \% \mathrm{PtC}$ catalyst in immunity against methanol crossover and long-term operation stability, thus, representing a promising metal-free electrocatalyst as cathode in alkaline fuel cell applications or micro fuel cells. The existing printing-based fabrication techniques of graphene materials provide favorable alternatives to conventional methods enabling clean, low-cost and facile large-scale production of nitrogen-doped graphene for electrochemical energy conversion.

\section{Acknowledgments}

We gratefully acknowledge the German Research Association (Deutsche Forschungsgesellschaft) for supporting this work within the framework of the Research Training Group "Micro Energy Harvesting" (Graduiertenkolleg 1322).

\section{References}

[1] Winter, M., and Brodd, R. J. 2004. "What Are Batteries, Fuel Cells and Supercapacitors?" Chem. Rev. 104 (10): 4245-70.

[2] Gasteiger, H. A., Kocha, S. S., Sompalli, B., and Wagner, F. T. 2005. "Activity Benchmarks and Requirements for Pt, Pt-Alloy, and Non-Pt Oxygen Reduction Catalysts for PEMFCs.” Applied Catalysis B: Environmental 56 (1-2): 9-35.

[3] Wang, B. 2005. "Recent Development of Non-platinum Catalysts for Oxygen Reduction Reaction." Journal of Power Sources 152 (1): 1-15.

[4] Zhu, C., and Dong, S. 2013. "Recent Progress in Graphene-Based Nanomaterials as Advanced 
Electrocatalysts towards Oxygen Reduction Reaction." Nanoscale 5 (5): 1753-67.

[5] Dreyer, D. R., Park, S., Bielawski, C. W., and Ruoff, R. S. 2010. "The Chemistry of Graphene Oxide." Chemical Society Reviews 39 (1): 228-40.

[6] Li, Y., and Wu, Y. 2009. "Coassembly of Graphene Oxide and Nanowires for Large-Area Nanowire Alignment." $J$. Am. Chem. Soc. 131 (16): 5851-7.

[7] Balandin, A. A., Ghosh, S., Bao, W., Calizo, I., Teweldebrhan, D., Miao, F., and Lau, C. N. 2008. "Superior Thermal Conductivity of Single-Layer Graphene." Nano Lett. 8 (3): 902-7.

[8] Novoselov, K. S., Geim, A. K., Morozov, S. V., Jiang, D., Katsnelson, M. I., Grigorieva, I. V., Dubonos, S. V., and Firsov, A. A. 2005. "Two-Dimensional Gas of Massless Dirac Fermions in Graphene.” Nature 438 (7065): 197-200.

[9] Meyer, J. C., Geim, A. K., Katsnelson, M. I., Novoselov, K. S., Booth, T. J., and Roth, S. 2007. "The Structure of Suspended Graphene Sheets.” Nature 446 (7131): 60-3.

[10] Stankovich, S., Dikin, D. A., Dommett, G. H. B., Kohlhaas, K. M., Zimney, E. J., Stach, E. A., Piner, R. D., Nguyen, S. T., and Ruoff, R. S. 2006. "Graphene-Based Composite Materials." Nature 442 (7100): 282-6.

[11] Qu, L., Liu, Y., Baek, J. B., and Dai, L. 2010. "Nitrogen-Doped Graphene as Efficient Metal-Free Electrocatalyst for Oxygen Reduction in Fuel Cells." ACS Nano 4 (3): 1321-6.

[12] Liang, J., Jiao, Y., Jaroniec, M., and Qiao, S. Z. 2012. "Sulfur and Nitrogen Dual-Doped Mesoporous Graphene Electrocatalyst for Oxygen Reduction with Synergistically Enhanced Performance." Angewandte Chemie 51 (46): 11496-500.

[13] Gong, K., Du, F., Xia, Z., Durstock, M., and Dai, L. 2009. "Nitrogen-Doped Carbon Nanotube Arrays with High Electrocatalytic Activity for Oxygen Reduction." Science 323 (5915): 760-4.

[14] Tang, Y., Allen, B. L., Kauffman, D. R., and Star, A. 2009. "Electrocatalytic Activity of Nitrogen-Doped Carbon Nanotube Cups.” J. Am. Chem. Soc. 131 (37): 13200-1.

[15] Wang, H., Maiyalagan, T., and Wang, X. 2012. "Review on Recent Progress in Nitrogen-Doped Graphene: Synthesis, Characterization, and Its Potential Applications." ACS Catal. 2 (5): 781-94.

[16] Geng, D., Chen, Y., Chen, Y., Li, Y., Li, R., Sun, X., Ye, S., and Knights, S. 2011. "High Oxygen-Reduction Activity and Durability of Nitrogen-Doped Graphene." Energy Environ. Sci. 4 (3): 760.

[17] Pan, F., Jin, J., Fu, X., Liu, Q., and Zhang, J. 2013. "Advanced Oxygen Reduction Electrocatalyst Based on Nitrogen-Doped Graphene Derived from Edible Sugar and Urea." ACS Applied Materials \& Interfaces 5 (21): 11108-14.
[18] Shao, Y., Zhang, S., Engelhard, M. H., Li, G., Shao, G., Wang, Y., Liu, J., Aksay, I. A., and Lin, Y. 2010. "Nitrogen-Doped Graphene and Its Electrochemical Applications." J. Mater. Chem. 20 (35): 7491.

[19] Novoselov, K. S., Geim, A. K., Morozov, S. V., Jiang, D., Zhang, Y., Dubonos, S. V., Grigorieva, I. V., and Firsov, A. A. 2004. "Electric Field Effect in Atomically Thin Carbon Films." Science 306 (5696): 666-9.

[20] Berger, C., Song, Z., Li, T., Li, X., Ogbazghi, A. Y., Feng, R., Dai, Z., Marchenkov, A. N., Conrad, E. H., First, P. N., and de Heer, W. A. 2004. "Ultrathin Epitaxial Graphite: 2D Electron Gas Properties and a Route toward Graphene-Based Nanoelectronics." J. Phys. Chem. B 108 (52): 19912-6.

[21] Kim, K. S., Zhao, Y., Jang, H., Lee, S. Y., Kim, J. M., Kim, K. S., Ahn, J. H., Kim, P., Choi, J. Y., and Hong, B. H. 2009. "Large-Scale Pattern Growth of Graphene Films for Stretchable Transparent Electrodes." Nature 457 (7230): 706-10.

[22] Tölle, F. J., Fabritius, M., and Mülhaupt, R. 2012. "Emulsifier-Free Graphene Dispersions with High Graphene Content for Printed Electronics and Freestanding Graphene Films.” Adv. Funct. Mater. 22 (6): 1136-44.

[23] Tschoppe, K., Beckert, F., Beckert, M., and Mülhaupt, R. 2014. "Thermally Reduced Graphite Oxide and Mechanochemically Functionalized Graphene as Functional Fillers for Epoxy Nanocomposites." Macromol. Mater. Eng. 2 (300): 140-52.

[24] Tuinstra, F. 1970. "Raman Spectrum of Graphite." $J$. Chem. Phys. 53 (3): 1126.

[25] Kudin, K. N., Ozbas, B., Schniepp, H. C., Prud'homme, R. K., Aksay, I. A., and Car, R. 2008. "Raman Spectra of Graphite Oxide and Functionalized Graphene Sheets." Nano Lett. 8 (1): 36-41.

[26] Dresselhaus, M. S., Jorio, A., Hofmann, M., Dresselhaus, G., and Saito, R. 2010. "Perspectives on Carbon Nanotubes and Graphene Raman Spectroscopy." Nano Lett. 10 (3): 751-8.

[27] Kurak, K. A., and Anderson, A. B. 2009. "Nitrogen-Treated Graphite and Oxygen Electroreduction on Pyridinic Edge Sites." J. Phys. Chem. C 113 (16): 6730-4.

[28] Ikeda, T., Boero, M., Huang, S. F., Terakura, K., Oshima, M., and Ozaki, J. 2008. "Carbon Alloy Catalysts: Active Sites for Oxygen Reduction Reaction.” J. Phys. Chem. C 112 (38): 14706-9.

[29] Zhang, J. 2008. PEM Fuel Cell Electrocatalysts and Catalyst Layers. London: Springer London.

[30] Bard, A. J., and Faulkner, L. R. 2001. Electrochemical Methods: Fundamentals and Applications. New York: Wiley. 